Journal of Neurology, Neurosurgery, and Psychiatry 1986;49:87-89

Short report

\title{
The response to isoniazid of action tremor in multiple sclerosis and its assessment using polarised light goniometry
}

\author{
DAVID A FRANCIS, ${ }^{*}$ DAVID GRUNDY, $\dagger$ JAMES R HERON ${ }^{*} \dagger$ \\ From the Department of Neurology, ${ }^{*}$ North Staffordshire Hospital Centre, Stoke-on-Trent, and Department \\ of Postgraduate Medicine, $\dagger$ University of Keele, Keele, Staffordshire, UK
}

SUMMARY Isonicotinic acid hydrazide (isoniazid) was evaluated in five patients as a treatment for the control of severe cerebellar action tremor occurring in multiple sclerosis. Oral doses of isoniazid BPC were increased every 2 weeks from $300 \mathrm{mg}$ to $1200 \mathrm{mg}$ daily over an 8 week period. Four patients reported considerable symptomatic benefit at doses ranging from $600 \mathrm{mg}$ to $900 \mathrm{mg}$ daily. Polarised light goniometry demonstrated a two to three-fold reduction of tremor in these patients when standard methods of clinical assessment showed only marginal improvement.

A recent clinical study has suggested an important role for isonicotinic acid hydrazide (isoniazid) in the treatment of action tremor in multiple sclerosis. ${ }^{1}$ The therapeutic efficacy of isoniazid, used in this context, has been evaluated clinically and subject to patient and observer bias. A pilot study was therefore undertaken to quantify the apparent beneficial effect of isoniazid in the control of action tremor in patients with multiple sclerosis using polarised light goniometry.

\section{Patients and methods}

The effect of isoniazid in controlling tremor was investigated in five multiple sclerosis patients with severe cerebellar dysfunction and in a stable phase of their disease. Patient clinical data are summarised in the table. Four of the five patients were receiving drug treatment for spasticity (baclofen). Haematological investigations and biochemistry including liver function were measured before entry to the trial and regularly during the trial period. Acetylator phenotype status was determined by the method described by Maher et al. ${ }^{2}$

Address for reprint requests: Dr DA Francis, Department of Clinical Neurology, Institute of Neurology, Queen Square, London WC1 3BG.

Received 11 December 1984 and in revised form 19 April 1985. Accepted 22 April 1985

\section{Trial of treatment}

The following regime was used: Treatment 1: Weeks 1 and 2, pyridoxine $100 \mathrm{mg}$ daily; Treatment 2: Weeks 3 and 4, isoniazid BPC $300 \mathrm{mg}$, pyridoxine $100 \mathrm{mg}$ daily; Treatment 3: Weeks 5 and 6, isoniazid BPC $600 \mathrm{mg}$, pyridoxine $100 \mathrm{mg}$ daily; Treatment 4: Weeks 7 and 8 , isoniazid BPC $900 \mathrm{mg}$, pyridoxine $100 \mathrm{mg}$ daily; Treatment 5: Weeks 9 and 10 , isoniazid BPC $1200 \mathrm{mg}$, pyridoxine $100 \mathrm{mg}$ daily.

Polarised light goniometry measurement of tremor was assessed before and after treatment and during alterations in the regime.

Clinical status (Kurtzke Disability Status $\mathrm{Score}^{3}$ ) was assessed at the same time as polarised light goniometry measurement and each patient contributed a personal assessment of his progress (scale 0 to 10 ) determined by ability to perform daily living activities.

\section{Polarised light goniometry}

This consists of a polarised light source illuminating two photosensitive transducers strapped to the upper arm and forearm of the seated subject. These in turn are connected to a module which measures limb displacement during a standard arm movement and converts it to an electrical signal recorded on magnetic tape. A wired metal target, attached to a light emitting diode was positioned in front of the subject at shoulder height. The diode was illuminated by the investigator and the subject extinguished the light by touching the target with a wired metal thimble on his index finger (completing an electrical circuit). The diode illumination time represented the duration of this movement. A normal subject performs this action with a smooth tremor-free movement, patients with cerebellar dysfunc- 
Table Details of patients

\begin{tabular}{llllll}
\hline Case & Sex & Age (years) & Classification & Clinical manifestations & $\begin{array}{l}\text { Duration } \\
\text { (years) }\end{array}$ \\
\hline 1 & F & 32 & Clinically definite & $\begin{array}{l}\text { Optic neuritis } \\
\text { Progressive paraparesis and cerebellar signs }\end{array}$ & 15 \\
2 & M & 32 & Clinically definite & $\begin{array}{l}\text { Optic neuritis } \\
\text { Progressive paraparesis and cerebellar signs }\end{array}$ & 9 \\
3 & F & 36 & Clinically definite & $\begin{array}{l}\text { Bilateral optic neuritis } \\
\text { Progressive paraparesis and cerebellar signs }\end{array}$ & 12 \\
4 & F & 51 & Clinically definite & $\begin{array}{l}\text { Subclinical optic neuropathy } \\
\text { Progressive paraparesis and cerebellar signs }\end{array}$ & 9 \\
5 & F & 25 & Clinically definite & $\begin{array}{l}\text { Relapsing and remitting cerebellar and } \\
\text { spinal signs }\end{array}$ & 3 \\
\hline
\end{tabular}

tion show delayed initiation, increased action duration and a superimposed terminal tremor $(3$ to $5 \mathrm{~Hz})$. The tremor component was isolated, averaged over several repeated actions and, after passage through an integrator, read out as "tremor units" from a digital voltmeter.

\section{Results}

Pyridoxine alone and pyridoxine with conventional doses of isoniazid (treatments 1 and 2) showed no significant benefit. When higher doses of isoniazid were used (treatment 3) there was subjective improvement in upper limb function in relation to eating, dressing and writing. Clinical assessment indicated only marginal improvement (1 unit, Kurtzke cerebellar functional scale). Subjective improvement continued on treatment 4 in each case. Further increment of isoniazid (treatment 5) was not associated with further improvement and three patients (slow isoniazid acetylators) experienced toxic effects (anorexia and nausea).

Haematological and biochemical monitoring in all patients was normal throughout the trial. When treatment was stopped improvement ceased and patients reverted to their initial degree of disability. In fast acetylators this occurred within 72 hours and there was a transient worsening beyond their pretreatment level of tremor (rebound effect). In slow acetylators, return to pretreatment dysfunction was delayed by 2 or 3 weeks without any evidence of rebound phenomena.

Tremor units measured by polarised light goniometry were standardised using five control subjects defining a "normal range" of 0.01 to 0.05 units (mean 0.028). The range in the pretreatment group was 0.10 to 1.00 (mean 0.38 ). Where benefit followed isoniazid therapy there was a two to three fold consistent reduction in tremor on goniometry (fig). In one patient (No 4) goniometry scores were improved prior to commencing isoniazid indicating a possible placebo effect.

\section{Discussion}

It has been suggested that the control of tremor by $\stackrel{\mathbb{Q}}{\Omega}$ isoniazid is a response to increased intracerebral GABA metabolism. ${ }^{4}$ Isoniazid is an inhibitor of $\vec{\circ}$ GABA aminotransferase, the first degradation enzyme in the GABA pathway. In Huntington's $\vec{\omega}$

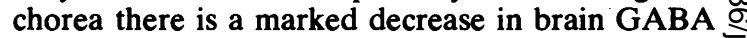
content. ${ }^{5}$ Although treatment with isoniazid is associated with an increase in CSF GABA levels, reports of the clinical value of this treatment in Hun- $\overrightarrow{0}$ tington's chorea are conflicting and uncertain. ${ }^{46}$ The situation in multiple sclerosis is analogous in th patients with chronic cerebral demyelination and 음 advanced cerebellar degeneration have also bee

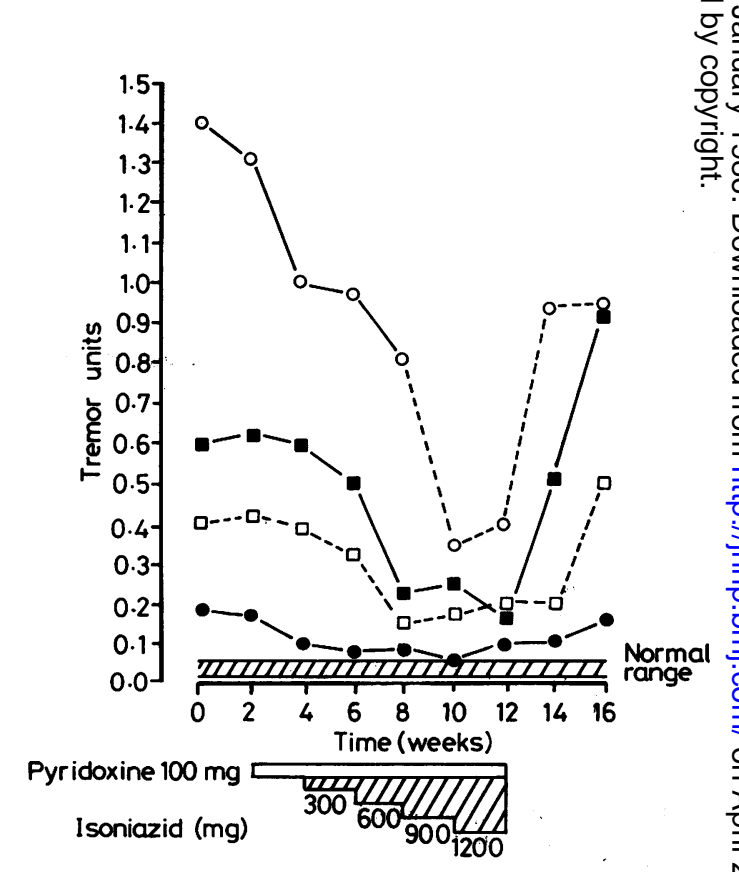

Fig Results of polarised light goniometry in four patients during the trial period (fifth patient withdrawn from trial). 
shown to have low CSF GABA levels. ${ }^{78}$ These patients could theoretically benefit from isoniazid therapy. As in Huntington's chorea established brain damage might be likely to modify the potential response.

Any of the suggested beneficial effect reported in this study could be explained on the basis of isoniazid's inhibitory action on monoamine oxidase rather than due to changes in GABA metabolism. This would apply to a general wellbeing and improved task performance. A further consideration must also be given to the possibility that isoniazid itself produces a therapeutic metabolite. In these circumstances, where high concentrations are achieved within the central nervous system the acetylator status of the patient may be important. There is evidence from our study that fast acetylators exhibit a rebound phenomenon following the abrupt withdrawal of the drug suggesting a fast metabolic turnover. This is in contrast to slow acetylators who exhibit side-effects on the same levels of treatments presumably as a result of reduced metabolite clearance.

Although there was improvement in tremor in this study it does not justify recommending isoniazid as treatment that is likely to be effective in the clinical management of the disabling tremor seen in multiple sclerosis. Further work is indicated in a larger controlled study and clearly analogues of isoniazid or its metabolites may prove more effective. When such trials are considered the use of polarised light goniometry is a potential method of objective assessment of response to treatment in a group of patients who are notoriously difficult to evaluate.

\section{References}

' Sabra AF, Hallett M, Sudarsky L, Mullally W. Treatment of action tremor with isoniazid. Neurology (NY) 1982;32:912-3.

${ }^{2}$ Maher JR, Whitney JM, Chambers JS, Stanonis DJ. The quantitative determination of isoniazid and paraamino-salicylic acid in body fluids. Am Rev Tuberc 1957;76:852-61.

${ }^{3}$ Kurtzke JF. Further notes on disability evaluation in multiple sclerosis, with scale modifications. Neurology (Minneap) 1965;15:654-61.

${ }^{4}$ Perry TL, Ulright JM, Hansen S, Macleod PM. Isoniazid therapy of Huntington disease. Neurology (Minneap) 1979;29:370-5.

${ }^{5}$ Perry TL, Hansen S, Kloster M. Huntingtons chorea: Deficiency of $\gamma$-aminobutyric acid in brain. $N$ Engl J Med 1973;288:337-42.

- McLean DR. Failure of isoniazid therapy in Huntington disease. Neurology (NY) 1982;32:1189-91.

7 Achar VS, Welch KMA, Chabi E, Bartosh K, Meyer JS. Cerebrospinal fluid gamma-aminobutyric acid in neurological disease. Neurology (Minneap) 1976; 26: 777-80.

${ }^{8}$ Kuroda H, Ogawa N, Yamamaki Y, et al. Cerebrospinal fluid GABA levels in various neurological and psychiatric diseases. J Neurol Neurosurg Psychiatry 1982;45:257-60. 